\section{Cancer grows on SOD1}

\section{By Michael J. Haas, Senior Writer}

A report in the Proceedings of the National Academy of Sciences shows how superoxide dismutase 1 acts as a master regulatory switch for kinase phosphorylation in angiogenesis and cell proliferation, making it a potential target to treat a variety of cancers. ${ }^{1}$ Although some mechanistic details remain to be elucidated, superoxide dismutase 1 inhibition could be used in combination with other cancer treatments and might have utility in some degenerative diseases as well.

Superoxide dismutase 1 (SOD1) is one of three SOD enzymes that catalyze the conversion of intracellular superoxide to hydrogen peroxide. Intracellular superoxide is produced when a growth factor binds to its receptor on the cell surface and induces receptor phosphorylation (see Figure 1, "Superoxide dismutase 1 in cancer").

Recently, SOD1 was identified as the target of tetrathiomolybdate (TTM), ${ }^{2}$ a compound that has antiangiogenic and antitumor activity in mice, ${ }^{3-5}$ but whose mechanism of action remained unclear.

In the PNAS paper, short interfering RNA and ATN-224, a second-generation TTM from Attenuon LLC, were used to study SOD1 inhibition in human umbilical vein endothelial cells (HUVECs) and multiple myeloma (MM) tumor cells.

The research team was led by Fernando Doñate while he was associate director of biology at Attenuon and included scientists from Cold Spring Harbor Laboratory and D.E. Shaw Research. Doñate is presently director of preclinical R\&D at Proacta Inc.

The researchers found that in proliferating cells, hydrogen peroxide oxidizes protein tyrosine phosphatases (PTPs) - such as PTP-1B - thus blocking their dephosphorylation activity. PTP inactivation in turn upregulates the kinase cascade, which ultimately drives phosphorylation of MAP kinase ERK-1 and MAP kinase ERK-2, both involved in angiogenesis and cell growth.

When SOD1 was inhibited, hydrogen peroxide levels decreased and PTPs remained active, resulting in reduced phosphorylation of ERK-1 and ERK-2.

"The kinases are still working, but the phosphatases win the battle," Doñate said. "This results in attenuation-not complete shutdown—of the phosphorylating kinase pathways."

Complete inhibition of SOD1 with ATN-224 induced apoptosis in most cell lines the team tested, but Doñate said they have not yet determined how this occurred.

Taken together, he said the results indicate ATN-224 could be complementary to tyrosine kinase inhibitor therapy for cancer. Such a strategy would both "hit the kinases and activate the phosphatases in tumors and endothelial cells," he said.

Previous studies by research teams at Wayne State University and Stanford University showed that SOD1 knockout mice are generally healthy, though they are more prone to liver cancer and some female mice have decreased fertility. 6,7

"The fact that SOD1 knockout mice have a mild phenotype supports the theory that resting cells can tolerate SOD1 being inhibited," making SOD1 a potentially attractive target in cancer cells, Doñate told SciBX.

Andrew Mazar, CSO at Attenuon and coauthor of the PNAS paper, told SciBX that ATN-224 is in four clinical trials: a Phase I/II trial in MM; Phase II trials for prostate cancer and metastatic melanoma, with data for both expected this year; and a Phase II trial in breast cancer, which began in April.

\section{New ground for SOD1}

Company representatives contacted by SciBX agreed that targeting levels of reactive oxygen species (ROS) like superoxide is a viable strategy for cancer, but differed in their opinions on whether SOD1 inhibition was the best way to do so.

"The PNAS paper shows an interesting new function for SOD1 that I don't think was appreciated until this was published," said Frank Bennett, SVP of research at Isis Pharmaceuticals Inc.

"It is becoming widely acknowledged that levels of ROS are elevated in a variety of acute and chronic inflammatory conditions including cancer, diabetes, neurodegenerative, renal and cardiovascular diseases," said Chris Wigley, VP of research at Reata Pharmaceuticals Inc.

Thus, Wigley said, it is encouraging to see work on therapeutic strategies that aim to influence ROS levels to modulate signaling pathways. "We anticipate the commercial development of such therapeutics will have dramatic implications for a vast array of inflammatory conditions," he said.

However, Wigley also suggested that targeting SOD1 to affect ROS levels might be too narrow a strategy given cancer's propensity to develop resistance to single-target therapies.

"A more advantageous approach to modulating ROS levels would be to increase cellular antioxidant capacity by inducing the production of a host of antioxidant enzymes and molecules," he said. "Such a general antioxidant approach to inhibiting ROS-mediated growth signaling is desirable, as opposed to selectively targeting a single enzyme," like SOD1, which decreases peroxide but increases superoxide.

Wigley added that a more general approach also would be desirable because of the tendency of SOD1 knockout mice to develop hepatic malignancies.

Reata’s RTA 402 takes just such a general approach. The compound is a synthetic triterpenoid that induces the anti-inflammatory transcription factor NF-E2-related factor 2 (Nrf2) and inhibits the transcriptional activities of NF- $\mathrm{KB}$ and signal transducer and activator of transcription 3 (STAT3).

RTA 402 is in two Phase I trials to treat advanced solid tumors or lymphoid malignancies. It is also in a Phase I/II trial to treat pancreatic cancer, with completion expected this year. 


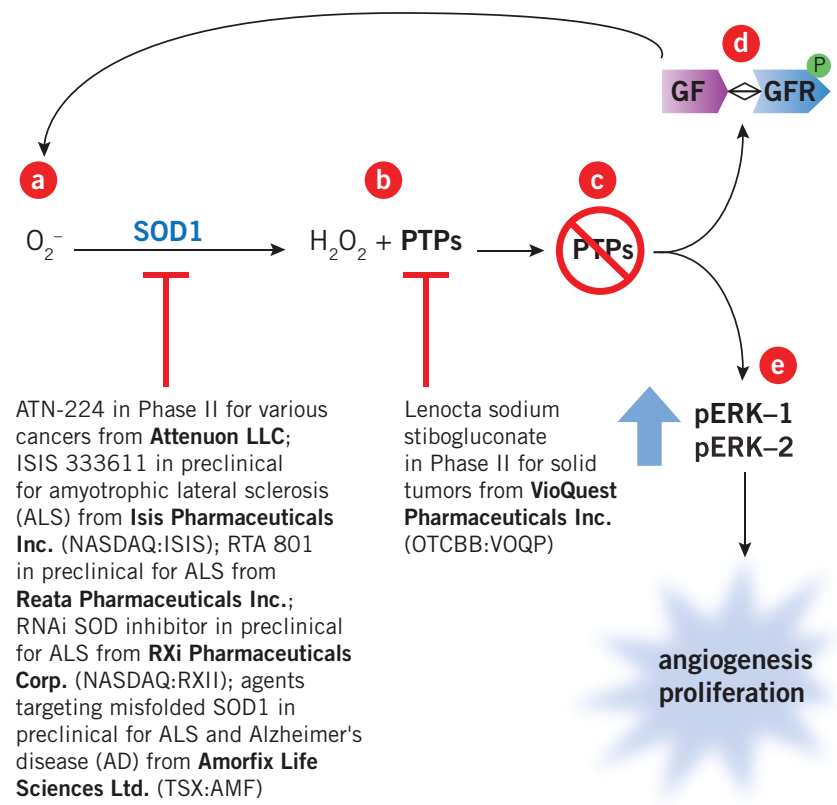

In April, Reata began a Phase II trial of the compound to treat diabetic nephropathy, and Wigley said the company plans to begin Phase II trials in additional inflammatory indications in $2 \mathrm{H} 08$.

Vernon Alvarez, VP of R\&D at VioQuest Pharmaceuticals Inc., said the results reported in PNAS show that SOD1 plays an important role in cancer. "But it is also evident that it needs to be completely abrogated in order to have full antiproliferative effects," he said.

VioQuest is developing Lenocta sodium stibogluconate, an inhibitor of Src homology PTPase-1 (SHP-1), SHP-2 and PTP-1B. It is in a Phase II trial in combination with interferon- $\alpha$ to treat advanced solid tumors, primarily melanoma and renal cell carcinoma (RCC).

Because Lenocta inactivates PTP-1B differently than ATN-224 does, "Lenocta may prove to be synergistic with SOD1 inhibition," Alvarez said. "Such a combination would be very interesting to study, he suggested, first for its effects on the expression of active PTP-1B and then in vitro and in vivo to determine whether the effect was synergistic.

"The role that PTPs play in angiogenesis is just beginning to be appreciated, but it is clear that many of the same PTPs are activated in angiogenesis" across tumor types, he said. "The impact of these PTPs to cancer cannot be overstated, but there is clearly much research to be done."

\section{ALSo applicable?}

SOD1 also has been implicated in amyotrophic lateral sclerosis (ALS). ${ }^{8}$

Isis's Bennett said ATN-224 is unlikely to provide any benefit in familial or inherited ALS, which comprises about $20 \%$ of all cases. A fraction of these familial cases are the result of a genetic mutation that causes SOD1 to fold abnormally, "without affecting its enzyme activity," he said. Instead, the misfolded SOD1 has abnormal protein-protein interactions, resulting in aggregates that characterize the disease.

Bennett said inhibiting SOD1 activity with a compound like ATN-224 is probably not useful for ALS because it does not remove the protein from the brain and therefore would not be expected to prevent the misfolded SOD1 from forming aggregates. "Instead, Isis aims to inhibit the expres-
Figure 1. Superoxide dismutase 1 in cancer. The elucidation of the role of superoxide dismutase 1 (SOD1) in angiogenesis and proliferation opens the door to new therapeutic approaches in cancer. Binding of growth factors (GFs; for example, epidermal growth factor, insulin-like growth factor-1 and fibroblast growth factor-2) to their respective receptors (GFRs) induces receptor phosphorylation, which produces superoxide $\left(\mathrm{O}_{2}^{-}\right)$by mechanisms not firmly established [a]. In the cytoplasm, superoxide is converted to hydrogen peroxide $\left(\mathrm{H}_{2} \mathrm{O}_{2}\right)$ through the superoxide dismutation reaction carried out by the catalyst SOD1 [b], and $\mathrm{H}_{2} \mathrm{O}_{2}$ then oxidizes protein tyrosine phosphatases (PTPs) such as PTP-1B. In turn, the dephosphorylation activity of PTPs is decreased [c], allowing the kinase cascade to proceed. This results in a feedback cycle that drives ongoing phosphorylation of GFRs [d] and upregulation of phosphorylated MAP kinase ERK-1 and phosphorylated MAP kinase ERK-2 (pERK-1; pERK-2) [e], leading to angiogenesis and cell proliferation.

sion of the SOD1 protein," he said.

But Bennett added that the PNAS paper did raise some questions worth investigating, such as whether the misfolded SOD1 can still bind ATN-224 and whether the compound's binding of misfolded SOD1 would prevent aggregation.

He said the latter is unlikely, but noted, "It's a prediction-and predictions can be wrong-so it's worth testing."

The company has ISIS 333611, an antisense oligonucleotide targeting SOD1, in preclinical toxicity studies. Isis plans to start a Phase I trial in familial ALS this year.

Reata's Wigley also said SOD1 inhibition would not necessarily be a benefit in ALS, noting that ATN-224 binds SOD1's copper ion, which is required for proper SOD1 structure and activity.

He said SOD1 without copper has been shown to be less structurally stable than it is with copper, and the structural instability of misfolded SOD1 in familial ALS correlates with disease severity.

Reata's RTA 801, a stabilizer that targets misfolded SOD1, is in preclinical development to treat ALS. Wigley said the company is working to identify additional SOD1 modulators for the disease.

Doñate agreed with both Bennett and Wigley that ATN-224 would probably have little use in ALS. "But ATN-224 could be useful in other degenerative diseases where copper is involved, such as Alzheimer's and Wilson's diseases," he said.

Over the last decade, a growing body of evidence has implicated copper ions in the formation of the amyloid plaques that characterize Alzheimer's disease $(\mathrm{AD})$, though the exact nature of that role is not fully

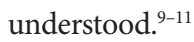

Until early this year, Pipex Pharmaceuticals Inc. was developing Coprexa, a first-generation oral TTM to treat Wilson's disease, an autosomal recessive genetic disorder characterized by the accumulation of copper in the brain, liver and other tissues. In January, however, the company received a refusal-to-file letter from the FDA for an NDA for the compound. 


\section{Unanswered questions}

The PNAS paper raises several follow-on questions relevant to cancer research, according to Doñate.

One is whether SOD1-mediated inhibition of growth factor receptor phosphorylation is sufficient to cause apoptosis. Doñate said, "We think not-that maybe something else is going on," but declined to elaborate.

Another question is what effect higher levels of superoxide-a consequence of SOD1 inhibition-have on the cell. He said the team presented data in the PNAS paper indicating some level of oxidative damage to the cell but added that "SOD1 knockdown suggests a tolerance for superoxide in nonproliferating cells."

He said the team had not investigated the contribution of higher superoxide levels to apoptosis in ATN-224-treated cancer cells.

A final question is how SOD1 inhibition downregulates expression of platelet-derived growth factor- $\beta$ receptor as reported in the paper.

Donate said the team has no immediate plans to continue investigating the SOD1 mechanism in cancer, but has collaborators with whom it might work on SOD1 in the future.

Proacta, Donate's current company, is developing hypoxia-activated prodrugs for use in combination with other chemotherapies. Attenuon, his former company, has filed a patent on the mechanism of SOD1 in cancer.

\section{RREFERENCES}

1. Juarez, J. et al. Proc. Natl. Acad. Sci. USA; published online May 12, 2008; doi:10.1073/pnas.0709451105

Contact: Fernando Doñate, Proacta Inc., San Diego, Calif. e-mail: fdonate@proacta.com

2. Juarez, J. et al. Clin. Cancer Res. 12, 4974-4982 (2006)

3. Pan, Q. et al. Cancer Res. 62, 4854-4859 (2002)

4. Pan, Q. et al. Mol. Cancer Ther. 1, 701-706 (2003)

5. Pan, Q. et al. Mol. Cancer Ther. 2, 617-622 (2003)

6. Ho, Y. et al. J. Biol. Chem. 273, 7765-7769 (1998)

7. Elchuri, S. et al. Oncogene 24, 367-380 (2005)

8. Osherovich, L. SciBX 1(8), 1-4; March 20, 2008

9. Opazo, C. et al. Biol. Res. 33, 125-131 (2000)

10. White, A. et al. J. Neurosci. 22, 365-376 (2002)

11. Price, K. et al. Recent Patents CNS Drug Discov. 2, 180-187 (2007)

\section{COMPANIES AND INSTITUTIONS MENTIONED}

Attenuon LLC, San Diego, Calif.

Cold Spring Harbor Laboratory, Cold Spring Harbor, N.Y.

D.E. Shaw Research, New York, N.Y.

Isis Pharmaceuticals Inc. (NASDAQ:ISIS), Carlsbad, Calif.

Pipex Pharmaceuticals Inc. (AMEX:PP), Ann Arbor, Mich.

Proacta Inc., San Diego, Calif.

Reata Pharmaceuticals Inc., Irving, Texas

Stanford University, Stanford, Calif.

VioQuest Pharmaceuticals Inc. (OTCBB:VOQP), Basking Ridge, N.J. Wayne State University, Detroit, Mich. 